\title{
On Definition and Evaluation of Mathematical Culture Attainment
}

\author{
Yinying Cai, Wei Zhang, Jia Tao \\ Department of Mathematics and Information Engineering, Chongqing University of Education, \\ Chongqing, 400067, China
}

Keywords: Mathematical culture, Mathematical culture attainment, Evaluation of mathematical culture

\begin{abstract}
This paper clarifies the concepts of "culture", "mathematical culture" and "mathematical culture attainment" and defines them again. Then, this paper discusses five elements of mathematical culture attainment and formulates ability classification standard of mathematical culture attainment by imitating PISA ability level.
\end{abstract}

\section{Definition of mathematical culture}

"Culture" is explained in Cihai as follows, "In a broad sense, culture refers to the sum of material and spritual production ability gained in social practice process, and created material and social practice. In a narrow sense, culture refers to spiritual production ability and spiritual products, including all social ideologies: natural science, technical science and social ideology.” This is the generalized culture. It is concept relative to nature. It refers to the sum of material wealth and spiritual wealth created for reform of natural state by human activities. In other words, all non-natural things or objects created by human beings are culture. Narrow culture refers to social ideology or concept form, i.e. people's spiritual life field.

Understanding of "culture" definition has a direct effect on the definition of "mathematical culture". Seeing from domestic and foreign research results, the definition of mathematical culture includes two directions. One of the directions is from the perspective of culture (i.e. definition of generalized culture). Mathematics is a kind of culture. In other words, mathematics is a sub-system of culture. The academician Li Daqian states mathematics is a kind of culture from multiple aspects such as knowledge, language, tool, science and technology. The professor Zhang Dianyu also believes that, "In specific social history, mathematical culture is the sum of national characteristics, traditional habits, rules and thinking ways shown when mathematics groups and individuals are engaged in mathematical activities. Colorful mathematical culture exists implicitly by taking symbolized, logical and formalized mathematical system as the carrier." ${ }^{\text {[1] }}$ Seeing from generalized definition of culture, mathematical knowledge hierarchy, language, thinking and spirit are a part of culture system.

The other direction is form the perspective of mathematics (i.e. narrow definition). Mathematics contains multiple ingredients of culture. In other words, mathematics and culture are two paratactic systems, but the two have fusion part. The professor Gu Pei from Nankai University considers, "mathematical culture also includes narrow sense and broad sense. Narrow mathematical culture refers to mathematical thought, spirit, method, view and language as well as their formation and development; generalized interpretation is that except these, mathematical culture also involves history of mathematics, mathematical beauty, mathematics education, cross of mathematics and humanity and relations between mathematics and various cultures." ${ }^{\text {,2] }}$ Seeing from narrow definition of culture, knowledge system of mathematics exists independently from culture system. Language, thinking and spirit of mathematics are a part of culture system.

No matter from which perspective mathematical culture is defined, knowledge system of mathematics is obviously the core of mathematical linguistics, mathematical thinking and mathematical spirit. Individuals may develop language competence, thinking ability and spiritual perception of mathematics through learning, experiencing, perceiving and re-thinking mathematical knowledge system. From the perspective of integrity of mathematical culture acquisition, it is more suitable to explain mathematical culture with generalized definition of culture. Mathematical culture 
defined from the perspective of generalized culture mostly takes into account of mathematical culture acquisition, but mathematical culture evaluation is seldom involved. This influences discussion of relations among mathematical culture, human society and individual life. ${ }^{[3]}$

In conclusion, mathematical culture is an organic whole, where mathematical culture system of mathematics serves as the center and relevant cultural fields such as the development, thought and spirit of mathematics serve as the important components. The basic elements of mathematics and various culture systems related to mathematics.

\section{Definition, nature and evaluation of mathematical culture attainment}

Attainment, i.e. culture and quality, refers to the skill and ability acquired by the subject in long-term social practice or after targeted training, or the level reached in thought, theory, knowledge and art. In combination of mathematical culture, mathematical culture attainment refers to basic quality that the subject cultivates in long-term social practice and especially mathematics-related practice (learning mathematics and applying mathematics) through experiencing, perceiving and re-thinking mathematical culture. Mathematical culture attainment is the ability of individuals to observe, cognize and appreciate problems from the view of mathematics based on existing mathematics experience and to judge, analyze and solve problems by use of mathematical thinking, knowledge and method and to communicate problems by use of mathematical language. ${ }^{[3]}$

\section{Five basic elements of mathematical culture attainment}

Mathematical culture attainment involves five basic elements: mathematical knowledge attainment, mathematical thinking attainment, mathematical language attainment, mathematical spirit attainment and mathematical application attainment. Mathematical knowledge attainment is individual mathematics level, i.e. individual knowledge level in elementary mathematics, axiomatic geometry, statistics, probability, infinitesimal calculus, modern algebra and other mathematics branches. In the process of learning mathematical knowledge, individuals can completely experience mathematical experience, perception and rethinking processes. The learning process of mathematical knowledge is an important approach to form mathematical culture attainment. Only when individuals own necessary mathematical knowledge attainment may they observe problems from the view of mathematics in social practice, solve problems with mathematical method and ponder over problems with mathematical thinking. Mathematical knowledge attainment is a basic precondition of mathematical knowledge attainment cultivation. Mathematical thinking attainment refers to individual abstract generalization capacity, spatial imagination ability, inferential capability and modeling ability etc. Mathematical language attainment manifests individual cognition, understanding and expression of symbols. Mathematical spirit attainment mainly shows reason, truth pursuit, innovation and cooperation. Mathematical application attainment refers to individual ability to handle practical problems with mathematical knowledge and skills in social practice. Other aspects of mathematical culture attainment are reflected by individuals through application of mathematics in real situations. Mathematical application attainment is a direct manifestation pattern of mathematical culture attainment. Individual mathematical knowledge attainment directly influences individual mathematical thinking, language, spirit perception and application ability. In turn, individual mathematical thinking attainment, language attainment, spirit attainment and application attainment facilitate individual cognition, learning and understanding of mathematical knowledge.

The precondition of mathematical culture attainment is mathematical knowledge attainment. Mathematical application attainment serves as explicit port, and the five aspects promote and influence each other.

\section{Nature of mathematical culture attainment}

Mathematical culture attainment owns individuality. Mathematical culture attainment integrates a series of psychology systems including experience, perception and introspection of mathematical culture. Even if different individuals appear in the same time and same space, their feelings are also 
different. Naturally, their experience, perception and introspection of mathematical culture are diverse, too. Thus, mathematical culture attainment with individual characteristics forms.

Mathematical culture attainment owns circumstance characteristic. The generation of any culture cannot be separated from specific historical environment and historical environment. If there is no "three mathematics crises", maybe there is no modern mathematics; if there was no "gambling game" which prevailed in the $17^{\text {th }}$ century, navigation cause with rapid development and insurance business, probability theory might not appear. The emergence of probability theory owns circumstance characteristic. Similarly, mathematical application also owns circumstance characteristic. Without realistic scenes of life, mathematical culture attainment cannot be embodied. Only in the specific scene, individuals can show mathematical culture attainment through mathematical application.

Mathematical culture attainment owns the characteristics of cultivation, stability, transcendence and quality. Mathematical culture attainment refers to individual attainment cultivated through experiencing, perceiving and rethinking mathematical culture. Mathematical need and application are inundated everywhere, ranging from shopping in the supermarket and space exploration. No matter whether individuals are willing, they will experience, perceive and rethink more or less. Naturally, they develop certain mathematical culture attainment. Once mathematical culture attainment is cultivated, it will exist in individual ideology for a long term. Just as Japanese Kunizo said, “After students graduate and enter the society, they almost have no opportunity to apply mathematical knowledge, so they will forget mathematical knowledge, soon within one or two years after their graduation. However, no matter what work they are engaged in, only mathematical spirit, thinking ways and research methods deeply engraved in brain will work anywhere and anytime and benefit them in their lifetime". Maybe individuals will forget mathematical culture knowledge, but they will continuously improve their mathematical culture attainment in the process of experiencing, perceiving and rethinking mathematical spirit, thinking, language and application.

Mathematical culture attainment owns explicitness. Once mathematical culture attainment is cultivated, individuals will automatically show it in real life. For example, before a child with the age of 5 does not master the addition, he will continuously ask for adults' opinions when they encounter relevant problems. However, once he masters the addition, relevant problems will be naturally solved by himself. We can judge whether individuals have mathematical culture attainment as long as individual behavior characteristics are observed in real situation.

Individuality of mathematical culture attainment indicates mathematical culture attainment can be studied. At least the differences of different individuals in mathematical culture attainment can be discussed. Cultivation and transcendence of mathematical culture attainment indicate of mathematical culture attainment can be cultivated through learning mathematical knowledge, experiencing, perceiving and rethinking mathematical culture. Circumstance characteristic, stability and explicitness of mathematical culture attainment show mathematical culture attainment can be observed. Individual mathematical culture attainment can be observed, analyzed and evaluated through setting the problems related to real situation.

\section{Evaluation of mathematical culture attainment}

To analyze and study individual mathematical culture attainment, there should be the method to observe mathematical culture attainment and evaluation standard. Seeing from the nature of mathematical culture attainment, mathematical culture attainment can be shown through individual handling of relevant problems in real situation. Hence, some problems closely related to real life can be set to observe and evaluate individual mathematical culture attainment through observing individual behavior characteristics and handling results during handling these problems. A direct way is to prepare a questionnaire containing the problems in real scene of life. Individual mathematical culture attainment can be observed and analyzed through analyzing questionnaire survey results.

Five elements of mathematical culture attainment are not mutually independent, but are an organic whole. Evaluation of mathematical culture attainment cannot be gained through independent assessment of each element. We need to a uniform evaluation method which can reflect the level of each element. 
PISA planned by OECD gives a similar evaluation method. PISA tests the ability of students at the age of 15 after compulsory education to apply knowledge and skills to solve realistic problems in reading, mathematics and science fields through questionnaire. PISA considers mathematical attainment not just requires students to master mathematical skills to adapt future social challenges, but also requires students to learn to analyze, reason and effectively convey ideas through proposing, analyzing and solving problems in various situations and fields. PISA gives the definition of mathematical attainment as follows: mathematical attainment is a kind of individual ability by which students can confirm and understand the functions of mathematics in society, gain mathematical judgment with sufficient basis and effectively apply mathematics. It is the mathematical ability which must be owned by a citizen which owns innovation spirit and thought, and concerns others in order to adapt current and future life. From 1997 till now, PISA has developed reliable and conventional evaluation index system. PISA divides students' mathematical attainment into 6 ability levels from high score to low score (refer to [4]). PISA test concerns students' ability to solve mathematical problems and apply mathematics in real situation. This has very high similarity with the test of mathematical culture attainment. By referring to PISA, individual mathematical culture attainment is divided into 6 ability levels from low score to high score.

Level 1: the individuals reaching Level 1 can solve familiar with obvious problems and proficiently apply calculator and other tools.

Level 2: the individuals reaching Level 2 can apply simple formulas and steps for 2-step reasoning and make literal explanation of results. Besides, they can extract relevant information from reference backgrounds and apply single representation symbols.

Level 3: the individuals reaching Level 3 can accurately and clearly execute the steps described in the background, select and apply simple problem solving strategies. Meanwhile, they can explain and use information expression methods acquired from different channels, conduct simple inference, interpret and exchange results.

Level 4: the individuals reaching Level 4 can establish models for specific situations with constraint conditions or need of some hypotheses, and choose different expression methods to correlate the results and practical problems. Besides, they can flexibly think and ratiocinate and form independent views and propositions.

Level 5: the individuals reaching Level 5 can propose problems and establish models in complex situations, and confirm constraint conditions and hypotheses. Furthermore, they can choose, select and evaluate solving strategies of corresponding problems. They not just can flexibly think, ratiocinate and express their views and propositions logically, but also rethink the results.

Level 6: the individuals reaching Level 6 can analyze and conclude problems of complex situations as well as establish models for problems of complex situations based on the survey. They can connect different information resources, carry out profound thinking and interference, flexibly apply symbols, operations and their mutual relations and form new methods and strategies in the new situations. In addition, they can accurately express their conclusions and findings, rethink and explain them and further optimize models.

\section{Acknowledgments}

Fund program: Humanistic and social science project of Chongqing Municipality Education Committee, Project name is Investigation and Research of Undergraduate Mathematical Culture Attainment. Project No.: 14skp04

\section{References}

[1] Zhang Dianyu, Mathematical culture. Ningbo: 2007 report of advanced training class of mathematics education under Ministry of Education, 2007

[2] Gu Pei, "Mathematical Culture" course and cultural quality education of college students. China University Teaching, 2007, (4). 
[3] Kang Shigang, Teaching research on mathematical attainment generation. Chongqing: Southwest University, 2009

[4] Wang lei, Evaluation of students' mathematical attainment by PISA. Shuxue Tongbao, 2009 (7) 